\title{
Quadratic Finite Volume Element Methods Based on Optimal Stress Points for Solving One-Dimensional Parabolic Problems
}

\author{
Sun Jia-hui ${ }^{1}$, Ma Ming-Juan ${ }^{2, a}$, Pang shi-chun ${ }^{3}$ Zhang Yong-po ${ }^{4}$ \\ ${ }_{1,2,3,4}$ Aviation university of air force, Changchun, Jilin Province ,china \\ aemail mingjuanma@gmail.com
}

Keywords: quadratic finite volume element method; parabolic equations; optimal stress points; error estimate.

Abstract. A new Lagrangian quadratic finite volume element method based on optimal stress points was presented for solving one-dimensional parabolic problem with trial and test spaces as the Lagrangian quadratic finite volume element space and the piecewise constant function space respectively. It is proved that the method has optimal order $\mathrm{H}^{1}$ and $\mathrm{L}^{2}$ error estimates. The numerical experiment confirms the results of theoretical analysis.

\section{Introduction}

The finite volume element method (FVEMS) is also called the generalized difference method (GDMS). The method that has a simple format structure, but also has the accuracy of the finite element method, and can keep the quantity of local conservation, it is an effective numerical method for solving partial differential equation of an effective numerical method, it has been widely used in computational fluid mechanics and electromagnetism, etc. At present there are a lot of research results for two-point boundary value problem of finite volume method. In the article, a new Lagrangian quadratic finite volume element method based on optimal stress points was presented for solving one-dimensional parabolic problem. It is proved that the method has optimal order $\mathrm{H}^{1}$ and $\mathrm{L}^{2}$ error estimates. The numerical experiment confirms the results of theoretical analysis.

\section{Finite volume element format}

Consider the mixed problem of one-dimensional parabolic equations on interval $\mathrm{I}=[\mathrm{a}, \mathrm{b}]$ :

$$
\left\{\begin{array}{cc}
\frac{\partial u}{\partial t}-\frac{\partial}{d x}\left(p \frac{\partial u}{\partial x}\right)=f(x, t), & (x, t) \in(a, b) \times(0, T] \\
u(a, t)=0, \frac{\partial u(b, t)}{\partial x}=0, & t \in(0, T] \\
u(x, 0)=u_{0}(x), & x \in(a, b)
\end{array}\right.
$$

Where $p \in C^{1}(I) ; p(x) \geq p_{\min }>0 ; f \in L^{2}(I)$. for convenience, recording $u_{t}=\frac{\partial u}{\partial t}, u^{\prime}=\frac{\partial u}{\partial x}$.

The associated weak formulation of problem (1) is:

find $u=u(\cdot, t) \in U:=H_{E}{ }^{1}(I)(0<t \leq T)$, such that

$$
\left\{\begin{array}{cc}
\left(u_{t}, v\right)+a(u, v)=(f, v), & \forall v \in U, 0<t \leq T, \\
u(x, 0)=u_{0}(x), & x \in(a, b)
\end{array}\right.
$$

where $(\cdot, \cdot)$ express the inner product of $L^{2}(I), a(u, v)=\int_{a}^{b} p u^{t} v^{t} d x$.

So the solution of the equations (2) is called generalized solution of the problem(1) let a division mesh $T_{h}$ for $I=[a, b]$, Nodes are $a=x_{0}<x_{1}<x_{2}<\cdots<x_{n}=b$. 
let $h_{i}=x_{i}-x_{i-1}, x_{i-1 / 2}=x_{i-1}+h_{i} / 2, i=1,2, \cdots, n, h=\max _{1 \leq i \leq n} h_{i}$,

And let the subdivision satisfy regularity conditions $h_{i} \geq \mu h(i=1,2, \cdots n), \mu$ is a positive number. Test space $U_{h}$ as the Lagrange quadratic finite element space corresponding to $T_{h}$. On the unit of $I_{i}=\left[x_{i-1}, x_{i}\right]$,

$$
\begin{aligned}
& u_{h}=u_{i-1}(2 \xi-1)+4 u_{i-1 / 2} \xi(1-\xi)+u_{i}(2 \xi-1) \xi= \\
& \left(\xi^{2}, \xi, 1\right)\left(\begin{array}{ccc}
2 & -4 & 2 \\
-3 & 4 & -1 \\
1 & 0 & 0
\end{array}\right)\left(\begin{array}{c}
u_{i-1} \\
u_{i-1 / 2} \\
u_{i}
\end{array}\right) \\
& u_{h}^{\prime}=u_{i-1}(4 \xi-3)+u_{i-1 / 2} \xi(-8 \xi+4) / h_{i}+u_{i}(4 \xi-1) \xi= \\
& (\xi, 1)\left(\begin{array}{cc}
-4 & 4 \\
3 & -1
\end{array}\right)\left(\begin{array}{c}
\left(u_{i-1 / 2}-u_{i-1}\right) / h_{i} \\
\left(u_{i}-u_{i-1 / 2}\right) / h_{i}
\end{array}\right) .
\end{aligned}
$$

where $\xi=\left(x-x_{i-1}\right) / h_{i}$

Then associated to $T_{h}$ dual mesh $T_{h}^{*}$,let $e_{1}=1 / 2-\sqrt{3} / 6, e_{2}=1 / 2+\sqrt{3} / 6$, then the two optimal stress points on interval $\left[x_{j-1}, x_{j}\right]$ is $x_{j-e_{1}}=x_{j}-e_{1} h_{j}, x_{j-e_{2}}=x_{j}-e_{2} h_{j}$, The dual unit node is

$$
a=x_{0}<x_{e_{1}}<x_{e_{2}}<\cdots x_{n-e_{2}}<x_{n-e_{1}}<x_{n}=b .
$$

Test function space $V_{h}$ take as piecewise constant function space corresponding to $T_{h}{ }^{*}$.suppose $\Pi_{h}{ }^{*}$ is the Interpolation of the projection operator form $U_{h}$ to $V_{h}$. $\prod_{h}$ is the Interpolation of the projection operator form $U$ to $U_{h}$, then

$$
\begin{gathered}
\left|\prod_{h} u-u\right|_{m} \leq C h^{2-m}|u|_{2}, m=0,1, \forall u \in U \\
\left|\prod_{h}^{*} u_{h}-u_{h}\right|_{0} \leq C h\left|u_{h}\right|_{1}, \forall u_{h} \in U_{h}
\end{gathered}
$$

Suppose $\mathrm{J}$ is a natural number, Time step is $\tau=T / \mathrm{J}$. define $g^{\theta}=g\left(x, t_{\theta}\right), t_{\theta}=\theta \tau$ for every function $g(x, t)$ on $\Omega \times[0, T]$. then define

$\partial_{t} g^{n}=\left(g^{n}-g^{n-1}\right) / \tau, g^{n-1 / 2}=\left(g^{n}+g^{n-1}\right) / 2$ for sequence $\left\{g^{n}\right\}_{n=0}^{J}$.

Then the Crank-Nicolson Fully discrete finite volume element format that Corresponding to problem (1) is request $u_{h}{ }^{n} \in U_{h}(n=1,2, \cdots, J)$, such that

$$
\left\{\begin{array}{cc}
\left(\partial_{t} u_{h}^{n}, v_{h}\right)+a\left(u_{h}^{n-1 / 2}, v_{h}\right)=\left(f^{n-1 / 2}, v_{h}\right), & \forall v_{h} \in V_{h} \\
u_{h}^{0}=u_{0 h}(x), & x \in(a, b)
\end{array}\right.
$$

Where $u_{0 h}$ is a discrete approximation of $u_{0}(x)$, This paper take approximation $\prod_{h} u_{0}$ for interpolation or Elliptic projection $R_{h} u_{0}$, then there has the following properties:

$$
\left\|u_{0}-u_{0 h}\right\|_{s} \leq C h^{r-s}, s=0,1, \quad 2 \leq r \leq 3 .
$$

\section{Error estimation}

Theorem 1 set $u$ is the solution of the problem (1) and $u_{h}^{n}$ is the solution of Fully discrete finite volume element format (8), then

1) 


$$
\begin{aligned}
\left\|u^{n}-u_{h}^{n}\right\|_{1} \leq C\left\{\left\|u_{0}-u_{0 h}\right\|_{1}+h^{2}\left\|u_{0}\right\|_{3}+h^{2} \int_{0}^{t_{n}}\left\|u_{t}\right\|_{3} d t+\right. \\
\left.h^{2}\left(\int_{0}^{t_{n}}\left\|u_{t}\right\|_{3}^{2} d t\right)^{1 / 2}+\tau^{2}\left(\int_{0}^{t_{n}}\left\|u_{t t t}\right\|_{0} d t\right)^{1 / 2}\right\}, n=0,1,2, \cdots, J ;
\end{aligned}
$$

2)

$$
\left\|u^{n}-u_{h}^{n}\right\|_{0} \leq C\left\{\left\|u_{0}-u_{0 h}\right\|_{0}+h^{3}\left\|u_{0}\right\|_{4}+h^{3} \int_{0}^{t_{n}}\left\|u_{t}\right\|_{4} d t+\tau^{2} \int_{0}^{t_{n}}\left\|u_{t t t}\right\|_{0} d t\right\}, n=0,1,2, \cdots, J
$$

3) If the initial value $u_{0 h}=\prod_{h} u_{0}$ or $R_{h} u_{0}$, then there has the following superconvergence estimates:

$$
\begin{aligned}
& \left\|u^{n}-\prod_{h} u^{n}\right\|_{1} \leq C\left(h^{3}+r^{2}\right), \\
& {\left[\frac{1}{r} \sum_{x_{0} \in N_{2}}\left|\left(u^{n}-u_{h}^{n}\right)^{\prime}\left(x_{0}\right)\right|^{2}\right]^{1 / 2} \leq C\left(h^{3}+\tau^{2}\right),}
\end{aligned}
$$

\section{Numerical experiments}

Consider parabolic problem:

$$
\left\{\begin{array}{cc}
\frac{\partial u}{\partial t}-\frac{\partial^{2} u}{\partial x^{2}}=0, & 0<x<\frac{\pi}{2}, 0<t \leq 1, \\
u(0, t)=0, \frac{\partial u}{\partial t} u\left(\frac{\pi}{2}, t\right)=0, & 0<t \leq 1 \\
u(x, 0)=\sin x, & 0<x<\frac{\pi}{2}
\end{array}\right.
$$

The exact solution of the problem (10) is $u(x)=e^{-1} \sin x$.

Comparing to the two methods show that the new method has a better convergence.

Table 1 Convergence orders of the method (8) for the problem (10)

\begin{tabular}{cllllll}
\hline $\mathrm{n}$ & $\left\|u^{n}-u_{h}^{n}\right\|_{0}$ & $\begin{array}{l}\text { Convergence } \\
\text { order }\end{array}$ & $\left\|u^{n}-u_{h}^{n}\right\|_{1}$ & $\begin{array}{l}\text { Convergence } \\
\text { order }\end{array}$ & $\left\|u_{h}^{n}-\prod_{h} u^{n}\right\|_{1}$ & $\begin{array}{l}\text { Convergence } \\
\text { order }\end{array}$ \\
\hline 8 & $2.4610 \times 10^{-5}$ & & $7.8417 \times 10^{-4}$ & & $1.0022 \times 10^{-5}$ & \\
16 & $2.9638 \times 10^{-6}$ & 3.0537 & $1.9381 \times 10^{-4}$ & 2.0165 & $1.0839 \times 10^{-6}$ & 3.2089 \\
32 & $3.6684 \times 10^{-7}$ & 3.0143 & $4.8315 \times 10^{-5}$ & 2.0041 & $1.2952 \times 10^{-7}$ & 3.0649 \\
64 & $4.5739 \times 10^{-8}$ & 3.0036 & $1.2070 \times 10^{-5}$ & 2.0010 & $1.5997 \times 10^{-8}$ & 3.0173 \\
128 & $5.7138 \times 10^{-9}$ & 3.0009 & $3.0170 \times 10^{-6}$ & 2.0003 & $1.9935 \times 10^{-9}$ & 3.0044 \\
\hline
\end{tabular}

Table 2 Convergence orders of usual quadratic FVEM for the problem (11)

\begin{tabular}{cllllll}
\hline $\mathrm{n}$ & $\left\|u^{n}-u_{h}^{n}\right\|_{0}$ & $\begin{array}{l}\text { Convergence } \\
\text { order }\end{array}$ & $\left\|u^{n}-u_{h}^{n}\right\|_{1}$ & $\begin{array}{l}\text { Convergence } \\
\text { order }\end{array}$ & $\left\|u_{h}^{n}-\prod_{h} u^{n}\right\|_{1}$ & $\begin{array}{l}\text { Convergence } \\
\text { order }\end{array}$ \\
\hline 8 & $1.0234 \times 10^{-4}$ & & $7.9049 \times 10^{-4}$ & & $1.0039 \times 10^{-4}$ & \\
16 & $2.6619 \times 10^{-5}$ & 1.9248 & $1.9561 \times 10^{-4}$ & 2.0147 & $2.6509 \times 10^{-5}$ & 1.9211 \\
32 & $6.7229 \times 10^{-6}$ & 1.9853 & $4.8780 \times 10^{-5}$ & 2.0037 & $6.7162 \times 10^{-6}$ & 1.9808 \\
64 & $1.6850 \times 10^{-6}$ & 1.9963 & $1.2187 \times 10^{-5}$ & 2.0009 & $1.6846 \times 10^{-6}$ & 1.9952 \\
128 & $4.2153 \times 10^{-7}$ & 1.9991 & $3.0463 \times 10^{-6}$ & 2.0002 & $4.2150 \times 10^{-7}$ & 1.9988 \\
\hline
\end{tabular}

\section{References}

[1] LI Rong-hua, CHEN Zhong-ying, WU Wei. Generalized Difference Methods for Differential Equations: Numerical Analysis of Minite Volume Element Methods [M].New York; Marcel Dekker,2000. 
[2] GAO Guang-hua,WANG Tong-ke. Cubic Superconvergent Finite Method for One-Dimensional Elliptic and Parabolic Equations [J].J Comput AppL Math,2009,233(9):2285-2301

[3]LI Yong-hai. A Generalized Difference Method for the Parabolic Equations [J]. Math Numersin,2002,24(4):487-500. 